\title{
Lorentz Invariance for Mixed Neutrinos
}

\author{
Massimo Blasone, \\ Dipartimento di Fisica and INFN, Università di Salerno, 84081 Baronissi (SA), Italy \\ João Magueijo, and Paulo Pires Pacheco \\ The Blackett Laboratory, Imperial College London, London SW7 2AZ, U.K.
}

Received on 23 January, 2005

\begin{abstract}
We show that a proper field theoretical treatment of mixed (Dirac) neutrinos leads to non-trivial dispersion relations for the flavor states. We analyze such a situation in the framework of the non-linear relativity schemes recently proposed by Magueijo and Smolin. We finally examine the experimental implications of our theoretical proposals by considering the spectrum and the end-point of beta decay in tritium.
\end{abstract}

\section{Introduction}

The subject of neutrino oscillations has now matured from an insightful prediction by Bruno Pontecorvo [1] and the early results of Homestake [2] to a structured framework backed by a wealth of new quantitative data [3-6]. This advances have been paralleled by much progress on the theoretical front with the efforts divided between phenomenological pursuits of more refined oscillation formulas and attempts to give the theory a sound formal structure within Quantum Field Theory (QFT).

A major outstanding question was that of the existence of a Hilbert space for the flavour states [7]. The Pontecorvo treatment of the latter in Quantum Mechanics (QM) actually turns out to be forbidden by the Bargmann super-selection rules [8]. This naturally pointed to QFT where the problem found its resolution [9-12]. Subsequently an even more consistent picture emerged with the discovery of an associated geometric phase [13], the extension to the case of threeflavours [14] and bosons [15, 16] and to the case of neutral fields [17]. The study of relativistic flavor currents [18, 19] was recently used to solve the phenomenologically very relevant problem of finding a space-oscillation formula [20].

Another important outcome of these studies is the understanding that the flavour eigenstates constitute the real physical entities, in contrast with the common view where the mass eigenstates are taken to be the fundamental objects [21].

The present paper proceeds in that direction by finding dispersion relations for the mixed neutrinos taking into account their nature as fundamental particles. We find that these dispersion relations no longer have the standard form thus exhibiting some form of breakdown of Lorentz invariance. This development is rather timely given the strong interest generated by various schemes involving such modifications [2227].

We further study the experimental implications of our analysis and compare it with the standard treatment, by considering the various possibilities which can arise in the end-point of the beta decay of tritium depending on which scenario turns out to be true.

The paper is organized as follows: In section 2 we show how flavor states can be properly defined in QFT. In section 3 we then consider the dispersion relations associated to such states. In section 4 we study the covariance of these forms and the description of the non-linear representation of the Poincaré algebra necessary to support them. Finally in section 5 we propose experimental tests, with special emphasis on the end-point of beta decay of tritium. Section 6 is devoted to conclusions.

\section{Flavor neutrino states in Quantum Field Theory}

Let us begin our discussion by considering the following Lagrangian density describing two free Dirac fields with a mixed mass term (see Appendix for conventions and further details):

$$
\mathcal{L}(x)=\bar{\Psi}_{f}(x)(i \not \partial-M) \Psi_{f}(x),
$$

where $\Psi_{f}^{T}=\left(\nu_{e}, \nu_{\mu}\right)$ and $M=\left(\begin{array}{cc}m_{e} & m_{e \mu} \\ m_{e \mu} & m_{\mu}\end{array}\right)$. The mixing transformations

$$
\begin{aligned}
& \nu_{e}(x)=\cos \theta \nu_{1}(x)+\sin \theta \nu_{2}(x) \\
& \nu_{\mu}(x)=-\sin \theta \nu_{1}(x)+\cos \theta \nu_{2}(x)
\end{aligned}
$$

with $\theta$ being the mixing angle, diagonalize the quadratic form of Eq.(1) to the Lagrangian for two free Dirac fields, with masses $m_{1}$ and $m_{2}$ :

$$
\mathcal{L}(x)=\bar{\Psi}_{m}(x)\left(i \not \partial-M_{d}\right) \Psi_{m}(x),
$$


where $\Psi_{m}^{T}=\left(\nu_{1}, \nu_{2}\right)$ and $M_{d}=\operatorname{diag}\left(m_{1}, m_{2}\right)$. One also has $m_{e}=m_{1} \cos ^{2} \theta+m_{2} \sin ^{2} \theta, m_{\mu}=m_{1} \sin ^{2} \theta+$ $m_{2} \cos ^{2} \theta, m_{e \mu}=\left(m_{2}-m_{1}\right) \sin \theta \cos \theta$. Without loss of generality we take $\theta$ ranging from 0 to $\frac{\pi}{4}$ (maximal mixing) and $m_{2}>m_{1}$.

The generator for the mixing relations (2) can be introduced as [9]:

$$
\begin{aligned}
\nu_{\sigma}(x) & \equiv G_{\theta}^{-1}(t) \nu_{j}(x) G_{\theta}(t) \\
G_{\theta}(t) & =\exp \left[\theta \int d^{3} \mathbf{x}\left(\nu_{1}^{\dagger}(x) \nu_{2}(x)-\nu_{2}^{\dagger}(x) \nu_{1}(x)\right)\right](5 .)
\end{aligned}
$$

with $(\sigma, j)=(e, 1),(\mu, 2)$ and $t \equiv x_{0}$. Note that $G_{\theta}(t)$ does not leave invariant the vacuum $|0\rangle_{1,2}$ :

$$
|0(t)\rangle_{e, \mu}=G_{\theta}^{-1}(t)|0\rangle_{1,2}
$$

We will refer to $|0(t)\rangle_{e, \mu}$ as to the flavor vacuum: it is orthogonal to $|0\rangle_{1,2}$ in the infinite volume limit [9]. We define the flavor annihilators, relative to the fields $\nu_{e}(x)$ and $\nu_{\mu}(x)$ as

$$
\begin{aligned}
\alpha_{\mathbf{k}, \sigma}^{r}(t) & \equiv G_{\theta}^{-1}(t) \alpha_{\mathbf{k}, j}^{r} G_{\theta}(t) \\
\beta_{-\mathbf{k}, \sigma}^{r \dagger}(t) & \equiv G_{\theta}^{-1}(t) \beta_{-\mathbf{k}, j}^{r \dagger} G_{\theta}(t)
\end{aligned}
$$

with $(\sigma, j)=(e, 1),(\mu, 2)$. The flavor fields can then be expanded in analogy to the free field case:

$$
\begin{aligned}
\nu_{\sigma}(x)= & \sum_{r=1,2} \int \frac{d^{3} \mathbf{k}}{(2 \pi)^{\frac{3}{2}}}\left[u_{\mathbf{k}, j}^{r}(t) \alpha_{\mathbf{k}, \sigma}^{r}(t)\right. \\
& \left.+v_{-\mathbf{k}, j}^{r}(t) \beta_{-\mathbf{k}, \sigma}^{r \dagger}(t)\right] e^{i \mathbf{k} \cdot \mathbf{x}} .
\end{aligned}
$$

with $(\sigma, j)=(e, 1),(\mu, 2)$.

The symmetry properties of the Lagrangian (1) have been studied in Ref.[18]: one has a total conserved charge $Q$ associated with the global $U(1)$ symmetry and time-dependent charges associated to the (broken) $S U(2)$ symmetry. Such charges are the relevant physical quantities for the study of flavor oscillations $[10,14]$. They are also essential in the definition of (physical) flavor neutrino states, as the one produced in a beta decay, for example.

In the present case of two flavors, we obtain for the flavor charges [18]:

$$
\begin{aligned}
& Q_{\sigma}(t)=\int d^{3} \mathbf{x} \nu_{\sigma}^{\dagger}(x) \nu_{\sigma}(x) \\
& =\sum_{r} \int d^{3} \mathbf{k}\left(\alpha_{\mathbf{k}, \sigma}^{r \dagger}(t) \alpha_{\mathbf{k}, \sigma}^{r}(t)-\beta_{-\mathbf{k}, \sigma}^{r \dagger}(t) \beta_{-\mathbf{k}, \sigma}^{r}(t)\right),
\end{aligned}
$$

with $\sigma=e, \mu$. By indicating with $Q_{j}(j=1,2)$, the (conserved) charge operators for the free fields, we obtain the following relations:

$$
\begin{aligned}
& Q_{\sigma}(t)=G_{\theta}^{-1}(t) Q_{j} G_{\theta}(t), \quad(\sigma, j)=(e, 1),(\mu, 2) \\
& \sum_{\sigma} Q_{\sigma}(t)=\sum_{j} Q_{j}=Q \quad ; \quad\left[Q, G_{\theta}(t)\right] \neq 0
\end{aligned}
$$

Thus the single neutrino and antineutrino states of definite flavor are defined in the following way:

$$
\begin{aligned}
& Q_{\sigma}(t)\left|\nu_{\sigma}^{\mathbf{k}}(t)\right\rangle=\left|\nu_{\sigma}^{\mathbf{k}}(t)\right\rangle \\
& Q_{\sigma}(t)\left|\bar{\nu}_{\sigma}^{\mathbf{k}}(t)\right\rangle=-\left|\bar{\nu}_{\sigma}^{\mathbf{k}}(t)\right\rangle
\end{aligned}
$$

and they naturally turn out to be vectors of the flavor Hilbert space $\mathcal{H}_{e, \mu}$ :

$$
\begin{aligned}
\left|\nu_{\sigma}^{\mathbf{k}}(t)\right\rangle & =\alpha_{\mathbf{k}, \sigma}^{r \dagger}(t)|0(t)\rangle_{e, \mu} \\
\left|\bar{\nu}_{\sigma}^{\mathbf{k}}(t)\right\rangle & =\beta_{\mathbf{k}, \sigma}^{r \dagger}(t)|0(t)\rangle_{e, \mu}
\end{aligned}
$$

One can also define the momentum operator for mixed fields [17]:

$$
\begin{aligned}
& \mathbf{P}_{\sigma}(t)=\int d^{3} \mathbf{x} \nu_{\sigma}^{\dagger}(x)(-i \nabla) \nu_{\sigma}(x) \\
& =\sum_{r} \int d^{3} \mathbf{k} \mathbf{k}\left(\alpha_{\mathbf{k}, \sigma}^{r \dagger}(t) \alpha_{\mathbf{k}, \sigma}^{r}(t)+\beta_{\mathbf{k}, \sigma}^{r \dagger}(t) \beta_{\mathbf{k}, \sigma}^{r}(t)\right)
\end{aligned}
$$

with

$$
\begin{aligned}
& \mathbf{P}_{\sigma}(t)=G_{\theta}^{-1}(t) \mathbf{P}_{j} G_{\theta}(t), \quad(\sigma, j)=(e, 1),(\mu, 2) \\
& \sum_{\sigma} \mathbf{P}_{\sigma}(t)=\sum_{j} \mathbf{P}_{j}=\mathbf{P} \quad ; \quad\left[\mathbf{P}, G_{\theta}(t)\right] \neq 0
\end{aligned}
$$

where $\mathbf{P}_{j}(j=1,2)$ are the (conserved) momentum operators for the free fields and $\mathbf{P}$ is the total momentum operator for the system (1), (3). It is immediate to verify that the flavor states Eq.(14),(15) have definite momentum (and helicity):

$$
\begin{aligned}
& \mathbf{P}_{\sigma}(t)\left|\nu_{\sigma}^{\mathbf{k}}(t)\right\rangle=\mathbf{k}\left|\nu_{\sigma}^{\mathbf{k}}(t)\right\rangle \\
& \mathbf{P}_{\sigma}(t)\left|\bar{\nu}_{\sigma}^{\mathbf{k}}(t)\right\rangle=\mathbf{k}\left|\bar{\nu}_{\sigma}^{\mathbf{k}}(t)\right\rangle .
\end{aligned}
$$

Note that the above defined flavor states differ from the ones commonly used which are defined by (erroneously) assuming that the Hilbert spaces for the flavor and the mass fields are the same. For further convenience we denote with a index " $P$ " the Pontecorvo flavor states:

$$
\begin{aligned}
& \left|\nu_{e}\right\rangle_{P}=\cos \theta\left|\nu_{1}\right\rangle+\sin \theta\left|\nu_{2}\right\rangle \\
& \left|\nu_{\mu}\right\rangle_{P}=-\sin \theta\left|\nu_{1}\right\rangle+\cos \theta\left|\nu_{2}\right\rangle
\end{aligned}
$$

for which we do not specify the momentum index: as it is well known [28], the flavor states so defined cannot have the same momentum or energy in all inertial frames. Note also that the states (21) are not eigenstates of the momentum and charge operators (defined in Eqs.(13) and (16)) as they are not the vectors of the flavor Hilbert space.

In the following, we will work in the Heisenberg picture, so the Hilbert space is chosen at the reference time $t=0$. We thus define our flavor states like

$$
\begin{aligned}
\left|\nu_{\sigma}^{\mathbf{k}}\right\rangle & \equiv \alpha_{\mathbf{k}, \sigma}^{r \dagger}(0)|0(0)\rangle_{e, \mu}, \\
\left|\bar{\nu}_{\sigma}^{\mathbf{k}}\right\rangle & \equiv \beta_{\mathbf{k}, \sigma}^{r \dagger}(0)|0(0)\rangle_{e, \mu}, \quad \sigma=e, \mu .
\end{aligned}
$$




\section{Dispersion relations for mixed neu- trinos}

Let us now consider the explicit expression for the one electron-neutrino state with definite helicity and momentum (at $t=0)$ :

$$
\begin{aligned}
\left|\nu_{e}^{\mathbf{k}}\right\rangle & =\prod_{\mathbf{k}} G_{\mathbf{k}}^{-1}(\theta) \alpha_{\mathbf{k}, 1}^{r \dagger}|0\rangle_{1,2}=\left[\cos \theta \alpha_{\mathbf{k}, 1}^{r \dagger}+\right. \\
& \left.+\left|U_{\mathbf{k}}\right| \sin \theta \alpha_{\mathbf{k}, 2}^{r \dagger}-\epsilon\left|V_{\mathbf{k}}\right| \sin \theta \alpha_{\mathbf{k}, 1}^{r \dagger} \alpha_{\mathbf{k}, 2}^{r \dagger} \beta_{\mathbf{k}, 1}^{r \dagger}\right] \times \\
& \times G_{\mathbf{k}, s \neq r}^{-1}(\theta) \prod_{\mathbf{p} \neq \mathbf{k}} G_{\mathbf{p}}^{-1}(\theta)|0\rangle_{1,2} .
\end{aligned}
$$

where we used $G_{\theta}(t)=\prod_{\mathbf{k}} G_{\mathbf{k}}(\theta)$. Eq.(23) shows clearly the non-trivial condensate structure of the flavor neutrino states in terms of the mass eigenstates.

Next we consider the energy-momentum tensor. For the massive fields $\nu_{j}$ we have:

$$
\mathcal{J}_{j}^{\mu \nu}(x) \equiv i \bar{\nu}_{j}(x) \gamma^{\nu} \partial_{\mu} \nu_{j}(x) \quad, \quad j=1,2
$$

from which the Hamiltonians for the free fields $\nu_{1}, \nu_{2}$ trivially follow:

$$
\begin{aligned}
& H_{j}=i \int d^{3} \mathbf{x} \nu_{j}^{\dagger}(x) \partial_{0} \nu_{j}(x) \\
& =\sum_{r} \int d^{3} \mathbf{k}\left(\alpha_{\mathbf{k}, j}^{r \dagger}(t) \partial_{0} \alpha_{\mathbf{k}, j}^{r}(t)+\beta_{\mathbf{k}, j}^{r}(t) \partial_{0} \beta_{\mathbf{k}, j}^{r \dagger}(t)\right) \\
& =\sum_{r} \int d^{3} \mathbf{k} \omega_{k, j}\left(\alpha_{\mathbf{k}, j}^{r \dagger} \alpha_{\mathbf{k}, j}^{r}-\beta_{\mathbf{k}, j}^{r} \beta_{\mathbf{k}, j}^{r \dagger}\right)
\end{aligned}
$$

with $j=1,2$. In a similar way we define the energymomentum tensor for the flavor fields:

$$
\mathcal{J}_{\sigma}^{\mu \nu}(x) \equiv i \bar{\nu}_{\sigma}(x) \gamma^{\nu} \partial_{\mu} \nu_{\sigma}(x) \quad, \quad \sigma=e, \mu .
$$

The energy operators are now:

$$
\begin{aligned}
& H_{\sigma}(t)=i \int d^{3} \mathbf{x} \nu_{\sigma}^{\dagger}(x) \partial_{0} \nu_{\sigma}(x) \\
& =\sum_{r} \int d^{3} \mathbf{k}\left(\alpha_{\mathbf{k}, \sigma}^{r \dagger}(t) \partial_{0} \alpha_{\mathbf{k}, \sigma}^{r}(t)+\beta_{\mathbf{k}, \sigma}^{r}(t) \partial_{0} \beta_{\mathbf{k}, \sigma}^{r \dagger}(t)\right)
\end{aligned}
$$

with $\sigma=e, \mu$. We also easily recover the momentum operators (16). Notice that Eq.(27) cannot be further reduced as for Eq.(25), due to the non-trivial time dependence of the flavor ladder operators.

In conclusion we find:

$$
\begin{aligned}
& H_{\sigma}(t) \neq G_{\theta}^{-1}(t) H_{j} G_{\theta}(t), \quad(\sigma, j)=(e, 1),(\mu, 2) \\
& \sum_{\sigma} H_{\sigma}(t)=\sum_{j} H_{j}=H \quad ; \quad\left[H, G_{\theta}(t)\right] \neq 0
\end{aligned}
$$

The inequality sign in (28) can be understood by noting the appearance of the time derivative in the definition (27) and the fact that the mixing generator is time dependent. Consequently, the result (29) is non-trivial and ensure the fact that the expectation value of the total (flavor field) energy on states in the flavor Hilbert space is time-independent.

We indeed have:

$$
\begin{aligned}
{ }_{e, \mu}\langle 0|H| 0\rangle_{e, \mu}= & -\int d^{3} \mathbf{k}\left(\omega_{k, 1}+\omega_{k, 2}\right) \times \\
& \times\left(1-2 \sin ^{2} \theta\left|V_{k}\right|^{2}\right)
\end{aligned}
$$

This has to be compared with the mass vacuum zero point energy:

$$
{ }_{1,2}\langle 0|H| 0\rangle_{1,2}=-\int d^{3} \mathbf{k}\left(\omega_{k, 1}+\omega_{k, 2}\right)
$$

The flavor vacuum zero-point energy has been studied in Ref.[29] in connection with the cosmological constant.

Of course, both contributions Eqs.(30), (31) are divergent and to properly define energy for flavor states, we need to normal order the Hamiltonian with respect to the relevant vacuum, namely the flavor vacuum:

$$
:: H: \equiv H-{ }_{e, \mu}\langle 0|H| 0\rangle_{e, \mu}
$$

where the new symbol for the normal ordering was introduced to remember that it refers to the flavor vacuum.

We finally obtain:

$$
\begin{aligned}
E_{e}(k) & \equiv\left\langle\nu_{e}^{\mathbf{k}}|: H::| \nu_{e}^{\mathbf{k}}\right\rangle \\
& =\omega_{k, 1} \cos ^{2} \theta+\left(1-2\left|V_{\mathbf{k}}\right|^{2}\right) \omega_{k, 2} \sin ^{2} \theta(33) \\
E_{\mu}(k) & \equiv\left\langle\nu_{\mu}^{\mathbf{k}}|: H::| \nu_{\mu}^{\mathbf{k}}\right\rangle \\
& =\omega_{k, 2} \cos ^{2} \theta+\left(1-2\left|V_{\mathbf{k}}\right|^{2}\right) \omega_{k, 1} \sin ^{2} \theta(34)
\end{aligned}
$$

We propose to treat these as modified dispersion relations and to find the corresponding non-linear realization of the Lorentz algebra as outlined in [25]. Obviously the energies in Eqs.(33),(34) are only expectation values subject to fluctuations but it is nevertheless sensible to consider the modified Lorentz transformation for these dispersion relations which form the classical limit of the theory.

Note the presence in the above dispersion relations, of the Bogoliubov coefficient $\left|V_{\mathbf{k}}\right|^{2}$ : this term is due to the flavor vacuum structure and is absent in the usual Pontecorvo case. The maximum of the function $\left|V_{\mathbf{k}}\right|^{2}$ occurs for $k_{\max }=$ $\sqrt{m_{1} m_{2}}$; we then have:

$$
\left|V_{k_{\max }}\right|^{2}=\frac{1}{2}-\frac{1}{\sqrt{\left(1+\frac{m_{1}}{m_{2}}\right)\left(1+\frac{m_{2}}{m_{1}}\right)}}
$$

If we put $a=\frac{m_{1}}{m_{2}}<1$, then the condition $\left|V_{\mathbf{k}}\right|^{2} \ll \frac{1}{2}$ is realized for

$$
1>a>\frac{b^{2}-1+2 b(1-\sqrt{2 b-1})}{(b-1)^{2}} \quad, \quad b \gg 1
$$

For example, for $b=100$ we get $a<0.75$ which is compatible with the current experimental bounds. This approximation was used in Ref.[30] where for simplicity we analyzed the dispersion relations obtained from the Pontecorvo states (21). In the following we treat the full case of Eqs.(33),(34). 


\section{Lorentz invariance for mixed neutri- nos}

In this section, we study the dispersion relations (33), (34) and derive the corresponding non-linear realization of the Lorentz algebra.

First, from Eqs.(33), (34), let us define the rest masses for the mixed neutrinos:

$$
\begin{aligned}
& m_{e} \equiv E_{e}(k=0)=m_{1} \cos ^{2} \theta+m_{2} \sin ^{2} \theta \\
& m_{\mu} \equiv E_{\mu}(k=0)=m_{2} \cos ^{2} \theta+m_{1} \sin ^{2} \theta
\end{aligned}
$$

Then we investigate the high $k$ limit of Eqs.(33),(34). To first order in $\frac{m_{j}^{2}}{2 k}$, it is $\omega_{k, j} \simeq k+\frac{m_{j}^{2}}{2 k}$ and $\left|V_{k}\right|^{2} \simeq \frac{\left(m_{2}-m_{1}\right)^{2}}{4 k^{2}}$ and we obtain:

$$
\begin{array}{ll}
E_{e}(k) \simeq k+\frac{\widetilde{m}_{e}^{2}}{2 k} ; & \widetilde{m}_{e}^{2} \equiv m_{1}^{2} \cos (2 \theta)+m_{1} m_{2} \sin ^{2} \theta \\
E_{\mu}(k) \simeq k+\frac{\widetilde{m}_{\mu}^{2}}{2 k} ; & \widetilde{m}_{\mu}^{2} \equiv m_{2}^{2} \cos (2 \theta)+m_{1} m_{2} \sin ^{2} \theta
\end{array}
$$

where we introduced the effective masses $\widetilde{m}_{e}$ and $\widetilde{m}_{\mu}$. We thus see that in the high momentum (or equivalently high $E$ since it is a monotonously growing function of $k$ ) limit, the dispersion relations for the flavor neutrinos are indeed of the usual form, although with a modified mass.

Noticing that $\omega_{k, 1}\left(1-2\left|V_{\mathbf{k}}\right|^{2}\right)=\frac{k^{2}+m_{1} m_{2}}{\omega_{k, 2}}$ and $\omega_{k, 2}(1-$ $\left.2\left|V_{\mathbf{k}}\right|^{2}\right)=\frac{k^{2}+m_{1} m_{2}}{\omega_{k, 1}}$, we rewrite Eqs.(33), (34) as

$E_{e}(k)=\frac{2 k^{2}+m_{1}\left(m_{2}+m_{1}\right)-m_{1}\left(m_{2}-m_{1}\right) \cos (2 \theta)}{2 \sqrt{k^{2}+m_{1}^{2}}}$

$E_{\mu}(k)=\frac{2 k^{2}+m_{2}\left(m_{2}+m_{1}\right)+m_{2}\left(m_{2}-m_{1}\right) \cos (2 \theta)}{2 \sqrt{k^{2}+m_{2}^{2}}}$

By introducing $a \equiv m_{2} / m_{1} \geq 1$, we get

$$
\begin{aligned}
& E_{e}(k)=\frac{k^{2}+m_{1}^{2}-(1-a) m_{1}^{2} \sin ^{2} \theta}{\sqrt{k^{2}+m_{1}^{2}}} \\
& E_{\mu}(k)=\frac{k^{2}+a^{2} m_{1}^{2}+a(1-a) m_{1}^{2} \sin ^{2} \theta}{\sqrt{k^{2}+a^{2} m_{1}^{2}}}
\end{aligned}
$$

It is easy to realize that, for $a>1$, the function $E_{\mu}(k)$ has an absolute minimum at $k=0$ with the value $E_{\mu}(0)=m_{\mu}$.

The situation is different for $E_{e}(k)$ : the minimum is now at $k_{\text {min }}=\frac{1}{\sqrt{2}} \sqrt{a-3+(1-a) \cos (2 \theta)}$. This is different from zero when $a$ is above the critical value $a_{c}=\frac{\cos (2 \theta)-3}{\cos (2 \theta)-1}$. For $1<a<a_{c}$, the function $E_{e}(k)$ has an absolute minimum at $k=0$ with the value $E_{e}(0)=m_{e}$.

This is represented in the two figures below for the case $\theta=\pi / 6 \Leftrightarrow a_{c}=5$.

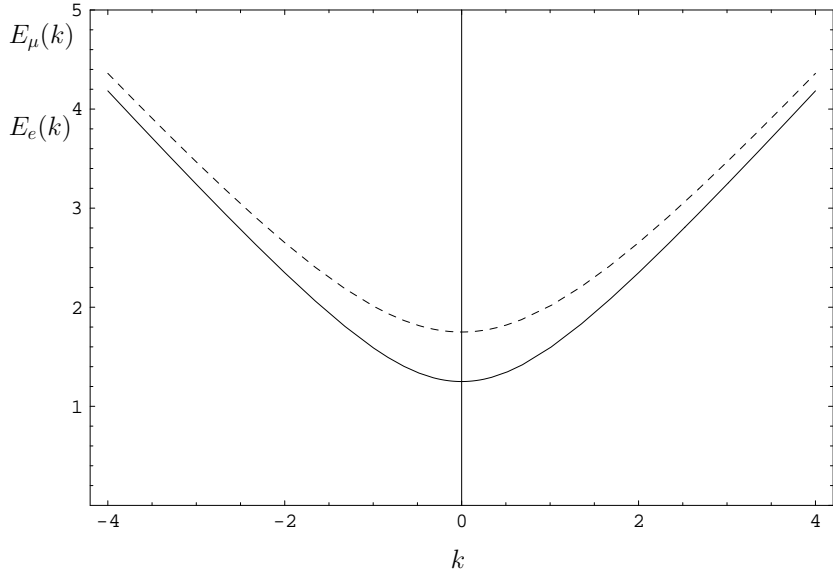

Figure 1. $E_{e}$ (solid line) and $E_{\mu}$ (dashed line) as functions of $k$ for $\theta=\pi / 6, m_{1}=1, a=2, a_{c}=5$.

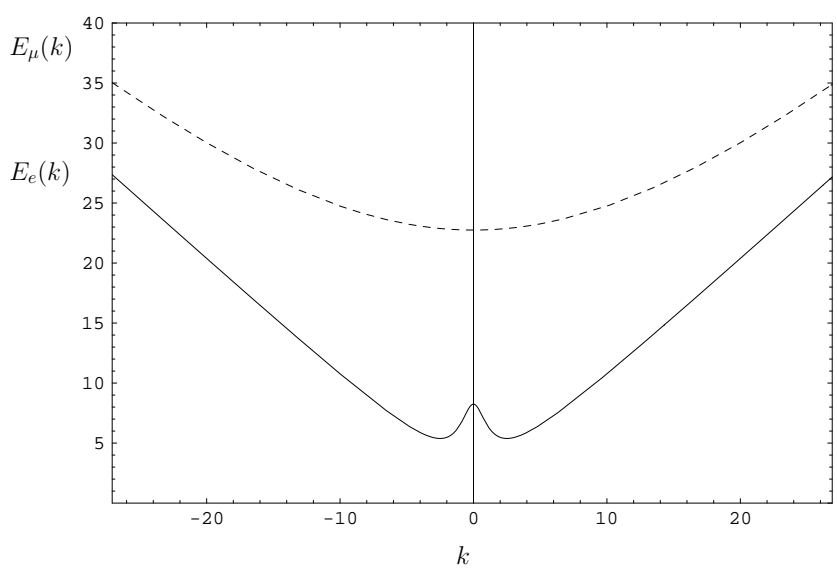

Figure 2. $E_{e}$ (solid line) and $E_{\mu}$ (dashed line) as functions of $k$ for $\theta=\pi / 6, m_{1}=1, a=30, a_{c}=5$.

In the following we consider only the subcritical case $a<a_{c}$. The case $a>a_{c}$ will be treated elsewhere.

Following Ref.[25], we now set the dispersion relations in the following form:

$$
\begin{aligned}
E_{e}^{2} f_{e}^{2}\left(E_{e}\right)-k^{2} g_{e}^{2}\left(E_{e}\right) & =m_{e}^{2} \\
E_{\mu}^{2} f_{\mu}^{2}\left(E_{\mu}\right)-k^{2} g_{\mu}^{2}\left(E_{\mu}\right) & =m_{\mu}^{2}
\end{aligned}
$$

It is now possible to identify the non-linear realization of the Lorentz group which leaves these dispersion relations invariant. They are generated by the transformation $U \circ(E, \mathbf{k})=(E f, \mathbf{k} g)$ applied to the standard Lorentz generators $\left(L_{a b}=p_{a} \frac{\partial}{\partial p^{b}}-p_{b} \frac{\partial}{\partial p^{a}}\right)$ :

$$
K^{i}=U^{-1}\left[p_{0}\right] L_{0}^{i} U\left[p_{0}\right] .
$$

This amounts to requiring linearity for the auxiliary variables $\tilde{E}=E f(E)$ and $\tilde{k}=k g(E)$. The resulting non-linear transformations for $E$ and $k$ are a non-linear representation of the Lorentz group ensuring that the deformed dispersion relations found for flavor states are valid in all frames. 
We find:

$$
\begin{aligned}
f_{e}^{2}\left(E_{e}\right) & =\frac{1}{2 E_{e}^{2}}\left[2 m_{e}\left(m_{2}-m_{1}\right) \sin ^{2} \theta\right. \\
& \left.+E_{e}\left(E_{e}+\sqrt{E_{e}^{2}-4 m_{1}\left(m_{2}-m_{1}\right) \sin ^{2} \theta}\right)\right] \\
g_{e}\left(E_{e}\right) & =1
\end{aligned}
$$

and

$$
\begin{aligned}
f_{\mu}^{2}\left(E_{\mu}\right) & =\frac{1}{2 E_{\mu}^{2}}\left[-2 m_{\mu}\left(m_{2}-m_{1}\right) \sin ^{2} \theta\right. \\
& \left.+E_{\mu}\left(E_{\mu}+\sqrt{E_{\mu}^{2}+4 m_{2}\left(m_{2}-m_{1}\right) \sin ^{2} \theta}\right)\right] \\
g_{\mu}\left(E_{\mu}\right) & =1
\end{aligned}
$$

It is easy to check that, for $m_{1}=m_{2}$ and/or $\theta=0$, we have $f_{e}^{2}\left(E_{e}\right)=f_{\mu}^{2}\left(E_{\mu}\right)=1$. Also $f_{\mu}^{2}\left(m_{\mu}\right)=1$ (for any $a \geq 1$ ) and $f_{e}^{2}\left(m_{e}\right)=1$ (only for $a_{c} \geq a \geq 1$ )

A plot of these two functions is given below:

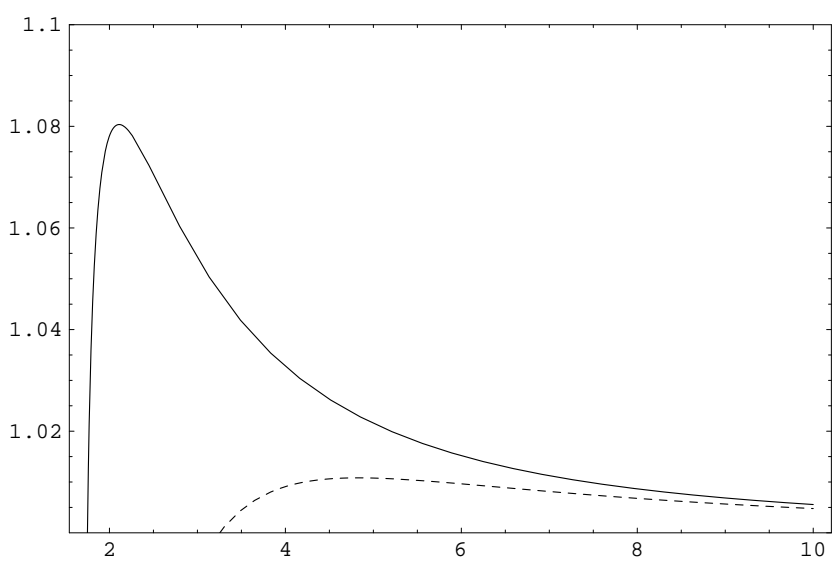

Figure 3. $f_{e}$ as a function of $E_{e}$ (solid line) and $f_{\mu}$ as a function of $E_{\mu}$ (dashed line) for $\theta=\pi / 6, m_{1}=1, a=4, a_{c}=5$.

For large values of $E_{e}$ and $E_{\mu}, f_{e}^{2}\left(E_{e}\right)$ and $f_{\mu}^{2}\left(E_{\mu}\right)$ can be approximated as:

$$
\begin{aligned}
& f_{e}^{2}\left(E_{e}\right) \simeq 1-\frac{1}{E_{e}^{2}}\left(m_{2}-m_{1}\right)^{2} \sin ^{4} \theta \\
& f_{\mu}^{2}\left(E_{\mu}\right) \simeq 1-\frac{1}{E_{\mu}^{2}}\left(m_{2}-m_{1}\right)^{2} \sin ^{4} \theta
\end{aligned}
$$

showing that the Lorentzian regime is approached quadratically as the energy (momentum) grows.

\section{Phenomenological consequences}

We now focus on some phenomenological consequences which arise from considering the flavor states as fundamental and consequently the non-standard dispersion relations (33), (34) as characterizing mixed neutrinos.
We consider the case of a beta decay process like tritium decay, which allows for a direct investigation of neutrino mass. In the following we take into account the various possible outcomes of this experiment in correspondence of the different theoretical possibilities for the nature of mixed neutrinos. We show that significative differences arise at phenomenological level between the standard theory and the scenario above described.

Let us then consider the decay:

$$
A \rightarrow B+e^{-}+\bar{\nu}_{e}
$$

where $A$ and $B$ are two nuclei (e.g. ${ }^{3} \mathrm{H}$ and ${ }^{3} \mathrm{He}$ ).

The electron spectrum is proportional to phase volume factor $E p E_{e} p_{e}$ :

$$
\frac{d N}{d K}=C E p(Q-K) \sqrt{(Q-K)^{2}-m_{e}^{2}}
$$

where $E=m+K$ and $p=\sqrt{E^{2}-m^{2}}$ are electron's energy and momentum. We denote by $m_{e}$ the electron (anti)neutrino mass.

The endpoint of $\beta$ decay is the maximal kinetic energy $K_{\max }$ the electron can take (constrained by the available energy $Q=E_{A}-E_{B}-m \approx m_{A}-m_{B}-m$ ). In the case of tritium decay, $Q=18.6 \mathrm{KeV}$. $Q$ is shared between the (unmeasured) neutrino energy and the (measured) electron kinetic energy $K$.

It is clear that if the neutrino were massless, then $m_{e}=0$ and $K_{\max }=Q$.

On the other hand, if the neutrino were a mass eigenstate (say with $m_{e}=m_{1}$ ), then $K_{\max }=Q-m_{1}$.

We now consider the various possibilities which can arise in the presence of mixing:

- If, following the common wisdom, mass eigenstates are considered fundamental, the $\beta$ spectrum is

$$
\frac{d N}{d K}=C E p E_{e} \sum_{j}\left|U_{e j}\right|^{2} \sqrt{E_{e}^{2}-m_{j}^{2}} \Theta\left(E_{e}-m_{j}\right)
$$

where $E_{e}=Q-K$ and $U_{e j}=(\cos \theta, \sin \theta)$ and $\Theta\left(E_{e}-m_{j}\right)$ is the Heaviside step function.

The end point is at $K=Q-m_{1}$ and the spectrum has an inflexion at $K \simeq Q-m_{2}$.

If flavor neutrinos are to be taken as fundamental, we have the following two options:

- Assuming that nuclei and the electron satisfy linear Lorentz transformations, and that $E_{e} f_{e}\left(E_{e}\right)$ transforms linearly, the only covariant law of energy conservation is

$$
E_{A}=E_{B}+E+E_{e} f_{e}\left(E_{e}\right) .
$$

The endpoint of $\beta$ decay is now $K_{\max }=Q-m_{e}$ and the $\beta$ spectrum is proportional to the phase volume factor $E p E_{e} f_{e}\left(E_{e}\right) p_{e}$ :

$$
\frac{d N}{d K}=C E p(Q-K) \sqrt{(Q-K)^{2}-m_{e}^{2}} \Theta\left(E_{e}-m_{e}\right)
$$




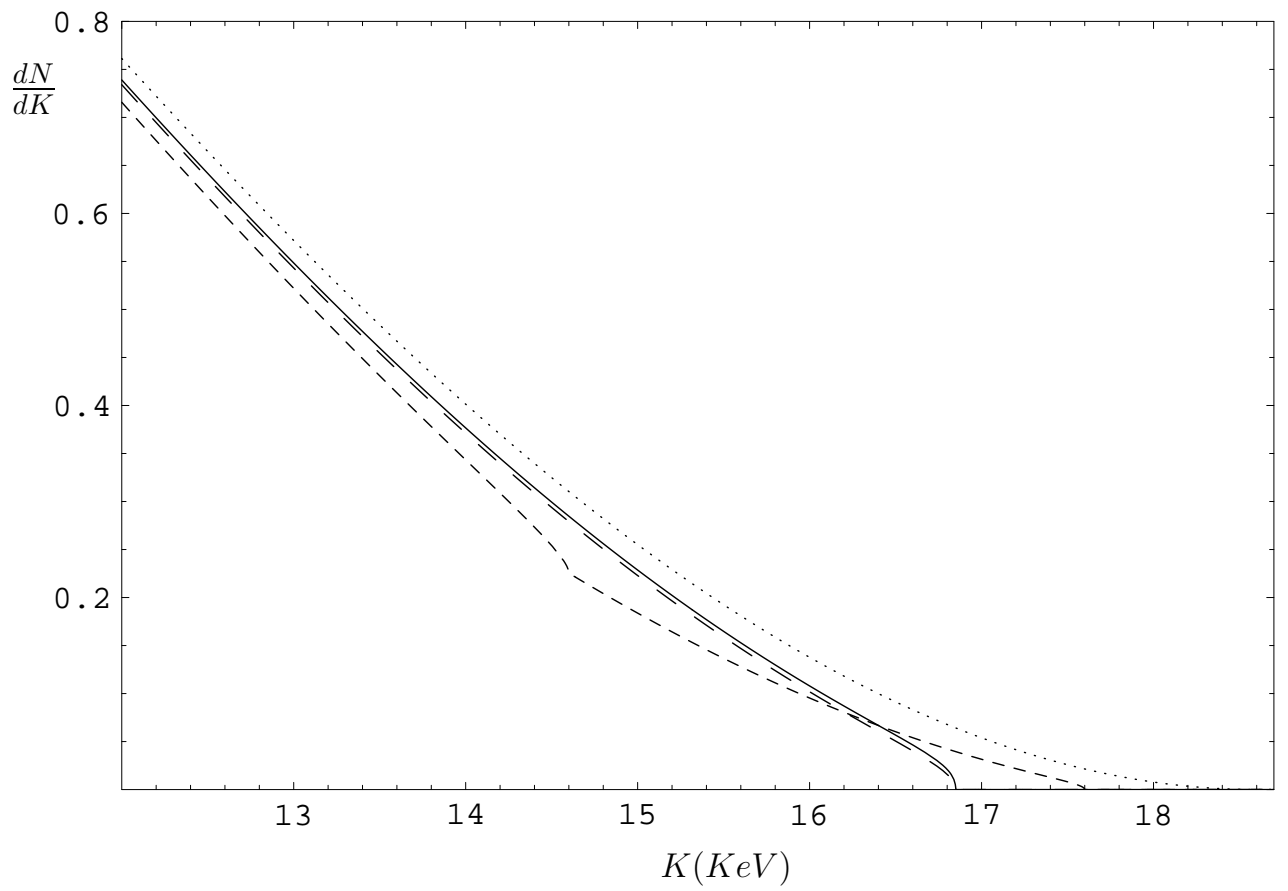

Figure 4. The tail of the tritium $\beta$ spectrum for: - a massless neutrino (dotted line); - a Lorentz invariant flavor state (solid line); - preferred frame (long-dashed line); - superposed prediction for 2 mass states (short-dashed line): notice the inflexion in the spectrum where the most massive state switches off. We used $m_{e}=1.75 \mathrm{KeV}, m_{1}=1 \mathrm{KeV}, m_{2}=4 \mathrm{KeV}, \theta=\pi / 6$.

- If, on the contrary, we insist upon the standard law

$$
E_{A}=E_{B}+E+E_{e}
$$

we have introduced a preferred frame, and are in conflict with the principle of relativity.

Then $K_{\max }=Q-m_{e}$ and the spectrum is proportional to the phase volume factor $E p E_{e} p_{e}$ :

$\frac{d N}{d K}=C E p(Q-K) \sqrt{(Q-K)^{2} f_{e}^{2}-m_{e}^{2}} \Theta\left(E_{e}-m_{e}\right)$

The above possibilities are plotted in Fig.(), together with the spectrum for a massless neutrino, for comparison.

We note that the next generation tritium beta decay experiments will allow a sub-eV sensitivity for the electron neutrino mass [31], thus hopefully allowing to unveil the true nature of mixed neutrinos.

\section{Conclusions}

In this paper, we have investigated some aspects of neutrino mixing in Quantum Field Theory. From a careful analysis of the Hilbert space structure for flavor (mixed) fields it has emerged that the flavor states, defined as eigenstates of the flavor charge, are at odds with Lorentz invariance. Indeed they exhibit non-standard dispersion relations, which however reduce to the usual (Lorentzian) ones in the relativistic limit.

We have then shown that it is possible to account for such a modified dispersion relations, by resorting to a recent proposal [25]: According to this, we could identify a non-linear representation of the Lorentz group allowing for these dispersion relations and ensuring at the same time the equivalence of inertial observers.

Finally, we have considered possible phenomenological consequences which can arise from our analysis, by looking at the beta decay. We have considered various possibilities, including that of introducing a preferred frame, and shown that observable differences arise in correspondence of the various cases.

\section{Acknowledgments}

M. B. acknowledges partial support from MURST, INFN, INFM and ESF Program COSLAB. P. P. P. thanks the FCT (part of the Portuguese Ministry of Education) for financial support under scholarship SFRH/BD/10889/2002.

\section{Appendix A: Flavor Hilbert space}

The free fields $\nu_{1}(x)$ and $\nu_{2}(x)$ are written as

$$
\begin{aligned}
\nu_{j}(x)= & \sum_{r=1,2} \int \frac{d^{3} \mathbf{k}}{(2 \pi)^{\frac{3}{2}}} e^{i \mathbf{k} \cdot \mathbf{x}}\left[u_{\mathbf{k}, j}^{r}(t) \alpha_{\mathbf{k}, j}^{r}\right. \\
& \left.+v_{-\mathbf{k}, j}^{r}(t) \beta_{-\mathbf{k}, j}^{r \dagger}\right], \quad j=1,2 .
\end{aligned}
$$

Here $u_{\mathbf{k}, j}^{r}(t)=e^{-i \omega_{k, j} t} u_{\mathbf{k}, j}^{r}$ and $v_{\mathbf{k}, j}^{r}(t)=e^{i \omega_{k, j} t} v_{\mathbf{k}, j}^{r}$, with $\omega_{k, j}=\sqrt{|\mathbf{k}|^{2}+m_{j}^{2}}$. 
The orthonormality and completeness relations are:

$$
\begin{aligned}
& u_{\mathbf{k}, j}^{r \dagger} u_{\mathbf{k}, j}^{s}=v_{\mathbf{k}, j}^{r \dagger} v_{\mathbf{k}, j}^{s}=\delta_{r s}, \\
& u_{\mathbf{k}, j}^{r \dagger} v_{-\mathbf{k}, j}^{s}=v_{-\mathbf{k}, j}^{r \dagger} u_{\mathbf{k}, j}^{s}=0, \\
& \sum_{r}\left(u_{\mathbf{k}, j}^{r} u_{\mathbf{k}, j}^{r \dagger}+v_{-\mathbf{k}, j}^{r} v_{-\mathbf{k}, j}^{r \dagger}\right)=\mathbb{I}_{2} .
\end{aligned}
$$

where $\mathbb{1}_{n}$ is the $n \times n$ unit matrix.

The $\alpha_{\mathbf{k}, j}^{r}$ and the $\beta_{\mathbf{k}, j}^{r}, j, r=1,2$ are the annihilation operators for the vacuum state $|0\rangle_{1,2} \equiv|0\rangle_{1} \otimes|0\rangle_{2}$ : $\alpha_{\mathbf{k}, j}^{r}|0\rangle_{12}=\beta_{\mathbf{k}, j}^{r}|0\rangle_{12}=0$.

The anticommutation relations are:

$$
\begin{aligned}
& \left\{\nu_{i}^{\alpha}(\mathbf{x}), \nu_{j}^{\beta \dagger}(\mathbf{y})\right\}_{t=t^{\prime}}=\delta^{3}(\mathbf{x}-\mathbf{y}) \delta_{\alpha \beta} \delta_{i j}, \alpha, \beta=1, . ., 4, \\
& \left\{\alpha_{\mathbf{k}, i}^{r}, \alpha_{\mathbf{q}, j}^{s \dagger}\right\}=\delta^{3}(\mathbf{k}-\mathbf{p}) \delta_{r s} \delta_{i j} \\
& \left\{\beta_{\mathbf{k}, i}^{r}, \beta_{\mathbf{q}, j}^{s \dagger}\right\}=\delta^{3}(\mathbf{k}-\mathbf{q}) \delta_{r s} \delta_{i j}, \quad i, j=1,2 .
\end{aligned}
$$

All other anticommutators are zero.

In the reference frame where $\mathbf{k}$ is collinear with $\hat{\mathbf{k}} \equiv$ $(0,0,1)$, the flavor annihilation operators have the simple form:

$$
\begin{aligned}
\alpha_{\mathbf{k}, e}^{r}(t)= & \cos \theta \alpha_{\mathbf{k}, 1}^{r}+\sin \theta\left(U_{\mathbf{k}}^{*}(t) \alpha_{\mathbf{k}, 2}^{r}\right. \\
& \left.+\epsilon_{\mathbf{k}}^{r} V_{\mathbf{k}}(t) \beta_{-\mathbf{k}, 2}^{r \dagger}\right) \\
\alpha_{\mathbf{k}, \mu}^{r}(t)= & \cos \theta \alpha_{\mathbf{k}, 2}^{r}-\sin \theta\left(U_{\mathbf{k}}(t) \alpha_{\mathbf{k}, 1}^{r}\right. \\
& \left.-\epsilon_{\mathbf{k}}^{r} V_{\mathbf{k}}(t) \beta_{-\mathbf{k}, 1}^{r \dagger}\right) \\
\beta_{-\mathbf{k}, e}^{r}(t)= & \cos \theta \beta_{-\mathbf{k}, 1}^{r}+\sin \theta\left(U_{\mathbf{k}}^{*}(t) \beta_{-\mathbf{k}, 2}^{r}\right. \\
& \left.-\epsilon_{\mathbf{k}}^{r} V_{\mathbf{k}}(t) \alpha_{\mathbf{k}, 2}^{r \dagger}\right) \\
\beta_{-\mathbf{k}, \mu}^{r}(t)= & \cos \theta \beta_{-\mathbf{k}, 2}^{r}-\sin \theta\left(U_{\mathbf{k}}(t) \beta_{-\mathbf{k}, 1}^{r}\right. \\
& \left.+\epsilon_{\mathbf{k}}^{r} V_{\mathbf{k}}(t) \alpha_{\mathbf{k}, 1}^{r \dagger}\right)
\end{aligned}
$$

where $\epsilon_{\mathbf{k}}^{r} \equiv(-1)^{r+\mathbf{k} \cdot \hat{\mathbf{k}}+1}$ and $U_{\mathbf{k}}(t), V_{\mathbf{k}}(t)$ are Bogoliubov coefficients given by:

$$
\begin{aligned}
U_{\mathbf{k}}(t) & \equiv u_{\mathbf{k}, 2}^{r \dagger}(t) u_{\mathbf{k}, 1}^{r}(t)=v_{-\mathbf{k}, 1}^{r \dagger}(t) v_{-\mathbf{k}, 2}^{r}(t) \\
& =\left|U_{\mathbf{k}}\right| e^{i\left(\omega_{k, 2}-\omega_{k, 1}\right) t}, \\
V_{\mathbf{k}}(t) & \equiv \epsilon_{\mathbf{k}}^{r} u_{\mathbf{k}, 1}^{r \dagger}(t) v_{-\mathbf{k}, 2}^{r}(t)=-\epsilon_{\mathbf{k}}^{r} u_{\mathbf{k}, 2}^{r \dagger}(t) v_{-\mathbf{k}, 1}^{r}(t) \\
& =\left|V_{\mathbf{k}}\right| e^{i\left(\omega_{k, 2}+\omega_{k, 1}\right) t}
\end{aligned}
$$

with

$$
\begin{aligned}
& \left|U_{\mathbf{k}}\right|=\frac{|\mathbf{k}|^{2}+\left(\omega_{k, 1}+m_{1}\right)\left(\omega_{k, 2}+m_{2}\right)}{2 \sqrt{\omega_{k, 1} \omega_{k, 2}\left(\omega_{k, 1}+m_{1}\right)\left(\omega_{k, 2}+m_{2}\right)}}, \\
& \left|V_{\mathbf{k}}\right|=\frac{\left(\omega_{k, 1}+m_{1}\right)-\left(\omega_{k, 2}+m_{2}\right)}{2 \sqrt{\omega_{k, 1} \omega_{k, 2}\left(\omega_{k, 1}+m_{1}\right)\left(\omega_{k, 2}+m_{2}\right)}}|\mathbf{k}| \\
& \left|U_{\mathbf{k}}\right|^{2}+\left|V_{\mathbf{k}}\right|^{2}=1 .
\end{aligned}
$$

\section{References}

[1] B. Pontecorvo, Zh. Eksp. Theor. Fiz. 33, 549 (1958); Sov. Phys. JEPT 6, 429 (1958); Z. Maki, M. Nakagawa, and
S. Sakata, Prog. Theor. Phys. 28, 870 (1962); V. Gribov and B. Pontecorvo, Phys. Lett. B 28, 493 (1969); S. M. Bilenky and B. Pontecorvo, Phys. Rep. 41, 225 (1978).

[2] J. Davis, D. S. Harmer, and K. C. Hoffmann, Phys. Rev. Lett. 20, 1205 (1968).

[3] M. Koshiba, in: *Erice 1998, From the Planck length to the Hubble radius* 170; S. Fukuda et al. (Super-Kamiokande collaboration), Phys. Rev. Lett. 86, 5656 (2001).

[4] Q. R. Ahmad et al. (SNO collaboration) Phys. Rev. Lett. 87, 071301 (2001); Phys. Rev. Lett. 89, 011301 (2002).

[5] K. Eguchi et al. (KamLAND Collaboration), Phys. Rev. Lett. 90, 021802 (2003).

[6] M. H. Ahn et al. (K2K Collaboration), [hep-ex/0212007].

[7] T. Kaneko, Y. Ohnuki, and K. Watanabe, Prog. Theor. Phys. 30, 521 (1963); K. Fujii, Nuovo Cimento 34, 722 (1964).

[8] V. Bargmann, Annals Math. 59, 1 (1954); A. Galindo and P. Pascual, Quantum Mechanics, (Springer Verlag, Berlin, 1990).

See also: D. M. Greenberger, Phys. Rev. Lett. 87, 100405 (2001).

[9] M. Blasone and G. Vitiello, Annals Phys. 244, 283 (1995); Erratum: ibid. 249, 363 (1995).

[10] M. Blasone, P. A. Henning, and G. Vitiello, Phys. Lett. B 451, 140 (1999); M. Blasone, in: *Erice 1998, From the Planck length to the Hubble radius* 584-593; M. Blasone and G. Vitiello, Phys. Rev. D 60, 111302 (1999).

[11] K. C. Hannabuss and D. C. Latimer, J. Phys. A 33, 1369 (2000); J. Phys. A 36, L69 (2003).

[12] K. Fujii, C. Hab, and T. Yabuki, Phys. Rev. D 59, 113003 (1999); Erratum: ibid. D 60, 099903 (1999); Phys. Rev. D 64, 013011 (2001); K. Fujii, C. Habe, and M. Blasone, [hep$\mathrm{ph} / 0212076]$.

[13] M. Blasone, P. A. Henning, and G. Vitiello, Phys. Lett. B 466, 262 (1999); X. B. Wang, L. C. Kwek, Y. Liu, and C. H. Oh, Phys. Rev. D 63, 053003 (2001).

[14] M. Blasone, A. Capolupo and G. Vitiello, Phys. Rev. D 66, 025033 (2002).

[15] M. Blasone, A. Capolupo, O. Romei, and G. Vitiello, Phys. Rev. D 63, 125015 (2001); M. Blasone, P. A. Henning, and G. Vitiello, in: "La Thuile 1996, Results and perspectives in particle physics" 139-152.

[16] M. Binger and C. R. Ji, Phys. Rev. D 60, 056005 (1999); C. R. Ji and Y. Mishchenko, Phys. Rev. D 64, 076004 (2001); Phys. Rev. D 65, 096015 (2002).

[17] M. Blasone and J. Palmer, Phys. Rev. D 69, 057301 (2004).

[18] M. Blasone, P. Jizba, and G. Vitiello, Phys. Lett. B 517, 471 (2001).

[19] M. Blasone, P. P. Pacheco, and H. W. Tseung, Phys. Rev. D 67, 073011 (2003)

[20] M. Beuthe, Phys. Rev. D 66, 013003 (2002); Phys. Rep. 375, $105(2003)$

[21] C. Giunti, [hep-ph/0409230].

[22] J. W. Moffat, Astrophys. Space Sci. 283, 505 (2003). 
[23] A. Albrecht and J. Magueijo, Phys. Rev. D 59, 043516 (1999).

[24] S. Alexander and J. Magueijo, [hep-th/0104093].

[25] J. Magueijo and L. Smolin, Phys. Rev. D 67, 044017 (2003); Phys. Rev. Lett. 88, 190403 (2002).

[26] D. Kimberly, J. Magueijo and J. Medeiros, Phys. Rev. D 70, 084007 (2004)

[27] G. Amelino-Camelia, Int. J. Mod. Phys. D 11, 35 (2002).
[28] C. Giunti, Mod. Phys. Lett. A 16, 2363 (2001).

[29] M. Blasone, A. Capolupo, S. Capozziello, S. Carloni, and G. Vitiello, Phys. Lett. A 323, 182 (2004); [hep-th/0412165].

[30] M. Blasone, J. Magueijo, and P. Pires-Pacheco, [hep$\mathrm{ph} / 0307205]$.

[31] A. Osipowicz et al. (KATRIN Collaboration), [hep-ex/0109033]. 